\title{
An individual measure of relative survival
}

\author{
Janez Stare, \\ University of Ljubljana, Slovenia \\ Robin Henderson \\ University of Lancaster, UK \\ and Maja Pohar \\ University of Ljubljana, Slovenia
}

[Received April 2003. Revised November 2003]

\begin{abstract}
Summary. Relative survival techniques are used to compare survival experience in a study cohort with that expected if background population rates apply. The techniques are especially useful when cause-specific death information is not accurate or not available as they provide a measure of excess mortality in a group of patients with a certain disease. Whereas these methods are based on group comparisons, we present here a transformation approach which instead gives for each individual an outcome measure relative to the appropriate background population. The new outcome measure is easily interpreted and can be analysed by using standard survival analysis techniques. It provides additional information on relative survival and gives new options in regression analysis. For example, one can estimate the proportion of patients who survived longer than a given percentile of the respective general population or compare survival experience of individuals while accounting for the population differences. The regression models for the new outcome measure are different from existing models, thus providing new possibilities in analysing relative survival data. One distinctive feature of our approach is that we adjust for expected survival before modelling. The paper is motivated by a study into the survival of patients after acute myocardial infarction.
\end{abstract}

Keywords: Myocardial infarction; Population mortality; Residual lifetime; Transformation models

\section{Introduction}

We start with a motivating example. In a study of survival of patients after acute myocardial infarction (AMI), which was carried out at the University Clinical Centre in Ljubljana, 1040 patients were followed for up to 14 years. The end point was death from any cause, as gathering of the cause-specific death information proved impossible to carry out, a situation that is quite common in such studies (see, for example, Dickman et al. (2004) for discussion). The patients were recruited from 1982 to 1986, with only those who were released from hospital included in the study. Many variables were recorded at the time of admission, but we first concentrate on sex and age only. A marginal analysis of the sex effect alone shows that women have significantly lower survival than men. This is well known and is usually explained by the fact that women are on the average older than men when AMI occurs. An analysis of our data set confirms this.

Address for correspondence: Janez Stare, Department of Medical Informatics, University of Ljubljana, Vrazov $\operatorname{trg}$ 2, SI-1000 Ljubljana, Slovenia.

E-mail: janez.stare@mf.uni-lj.si 
Table 1. Hazard ratio for sex (women versus men)

\begin{tabular}{|lcc|}
\hline Model includes & Hazard ratio for sex & p-value \\
\hline Sex & 1.7 & 0.000 \\
Sex and age & 1.1 & 0.364 \\
\hline
\end{tabular}

Table 1 shows estimated hazard ratios and $p$-values for sex in two Cox proportional hazards models, the first containing sex as the only explanatory variable, and the second also including age.

After the introduction of age the effect of sex disappears, meaning that, conditional on age, sex is apparently not related to survival. However, the story changes if we consider survival relative to the background population. The cumulative relative survival function is defined (e.g. Ederer et al. (1961)) as

$$
r(t)=S_{\mathrm{O}}(t) / S_{\mathrm{P}}(t)
$$

where $S_{\mathrm{O}}(t)$ denotes observed survival and $S_{\mathrm{P}}(t)$ stands for population or expected survival, which is estimated on the basis of life-tables. Here $t$ is the residual lifetime following AMI and both $S_{\mathrm{O}}(t)$ and $S_{\mathrm{P}}(t)$ are conditional on survival to age at AMI, although this is not made explicit in the notation.

Although the expected survival is, in principle, a simple concept, there are some problems in calculating it in a way that controls for heterogeneity in the observed follow-up times and making it independent of the observed mortality at the same time. Trying to avoid these pitfalls, several methods (Ederer et al., 1961; Ederer and Heise, 1959; Hakulinen, 1982) have been proposed for the calculation of $S_{\mathrm{P}}(t)$. It is widely accepted that in the absence of covariates the best approach is that of Hakulinen, which we used to obtain the $r(t)$ estimates that are shown in Fig. 1. Clearly, the relative survival of women is worse than that of men. This result can be easily explained. We have shown above that the survival of women after AMI, given age, is no different from the survival of men. However, the expected lifespan of women in the background population is longer than men's, and this is reflected in the relative survival.

The cumulative relative survival function $r(t)$ provides a useful summary of the survival experience of a cohort relative to the background population. In this paper we propose an alternative approach based on a simple transformation which provides a measure of survival relative to the background for each individual, without the need for grouping. The transformed survival time can be considered as the outcome variable of interest, summary statistics and plots are easily interpreted and the transformed variable can be taken directly as the response variable in regression modelling to investigate covariate effects. The transformation approach is described in Section 2 and its advantages as a direct relative survival measure are discussed in Section 3. Modelling to investigate covariate effects is described in Section 4 and related in Section 5 to existing methods for the regression analysis of survival data adjusted for background information. Some comments in Section 6 conclude the paper. Throughout, the timescale of interest is the residual lifetime $t$ following a clinical event such as AMI or a diagnosis of cancer.

The data that are analysed in the paper can be obtained from

http: / / www.blackwellpublishing.com/rss 


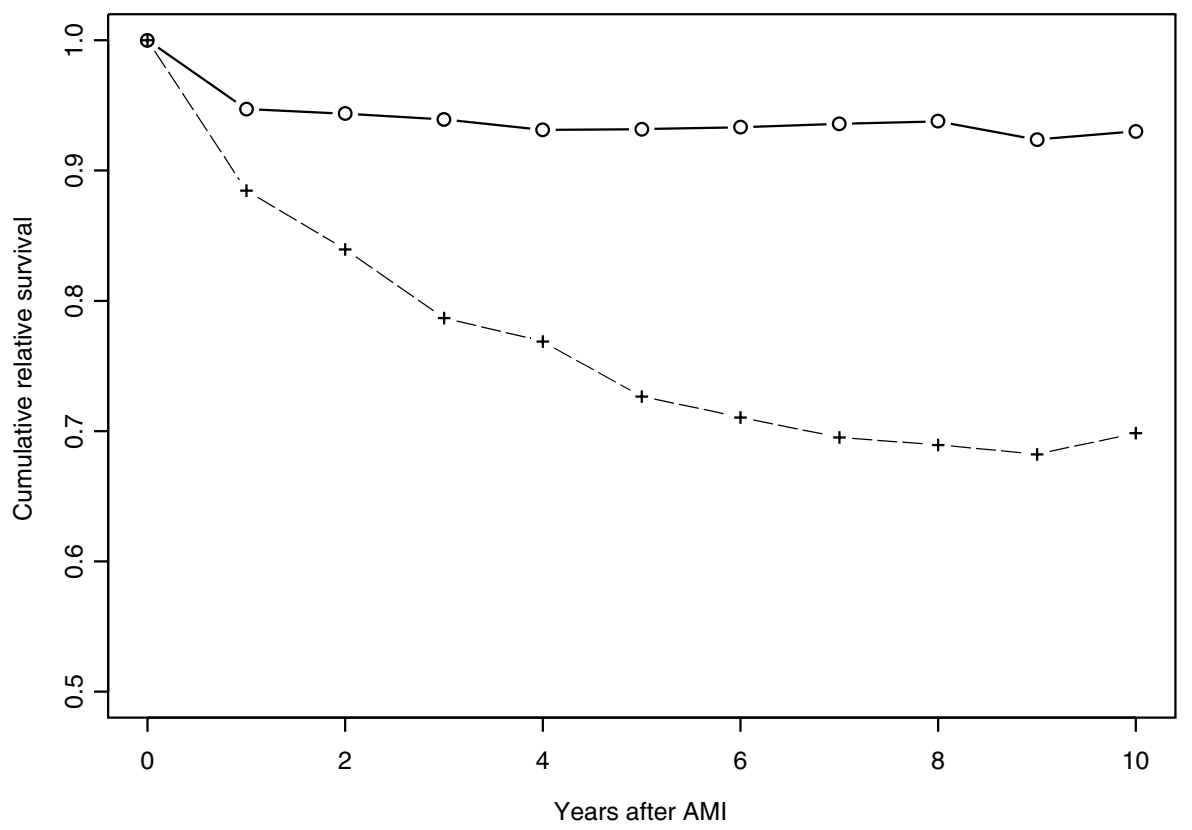

Fig. 1. Cumulative relative survival by sex: - , men; - - - women

Table 2. Example of population mortality data for the follow-up of men and women aged 63 years in the year 1986

\begin{tabular}{|lllllllll|}
\hline Year & Men & Women & Year & Men & Women & Year & Men & Women \\
\hline 1986 & 0.0286 & 0.0119 & 1991 & 0.0410 & 0.0169 & 1996 & 0.0503 & 0.0269 \\
1987 & 0.0323 & 0.0134 & 1992 & 0.0481 & 0.0196 & 1997 & 0.0571 & 0.0271 \\
1988 & 0.0334 & 0.0166 & 1993 & 0.0416 & 0.0246 & 1998 & 0.0695 & 0.0365 \\
1989 & 0.0325 & 0.0140 & 1994 & 0.0488 & 0.0237 & 1999 & 0.0600 & 0.0350 \\
1990 & 0.0338 & 0.0156 & 1995 & 0.0545 & 0.0258 & & & \\
\hline
\end{tabular}

\section{The outcome measure}

Relative survival methods are based on population mortality tables. These contain probabilities of dying for people of given sex and age in a given year. Table 2 gives examples from Slovenian mortality data for the follow-up of males and females, aged 63 years in the year 1986, showing how mortality rates vary as the individuals age.

From such tables we can easily calculate the conditional cumulative distribution function (CDF) for residual lifetime after any given age. Fig. 2 shows CDFs for the two examples from Table 2. Obviously, women do better than men. The two CDFs are truncated after 13 years follow-up, age 76 years, as the population tables were available only until the year 1999 . This is a common situation in relative survival analysis, but it presents no problem for the methods to be described in this paper as observation times also do not extend beyond the time for which the population tables are produced.

The basic idea in relative survival is to calculate the probability of surviving a certain period (usually yearly intervals) for the subjects in the sample, assuming that background population 


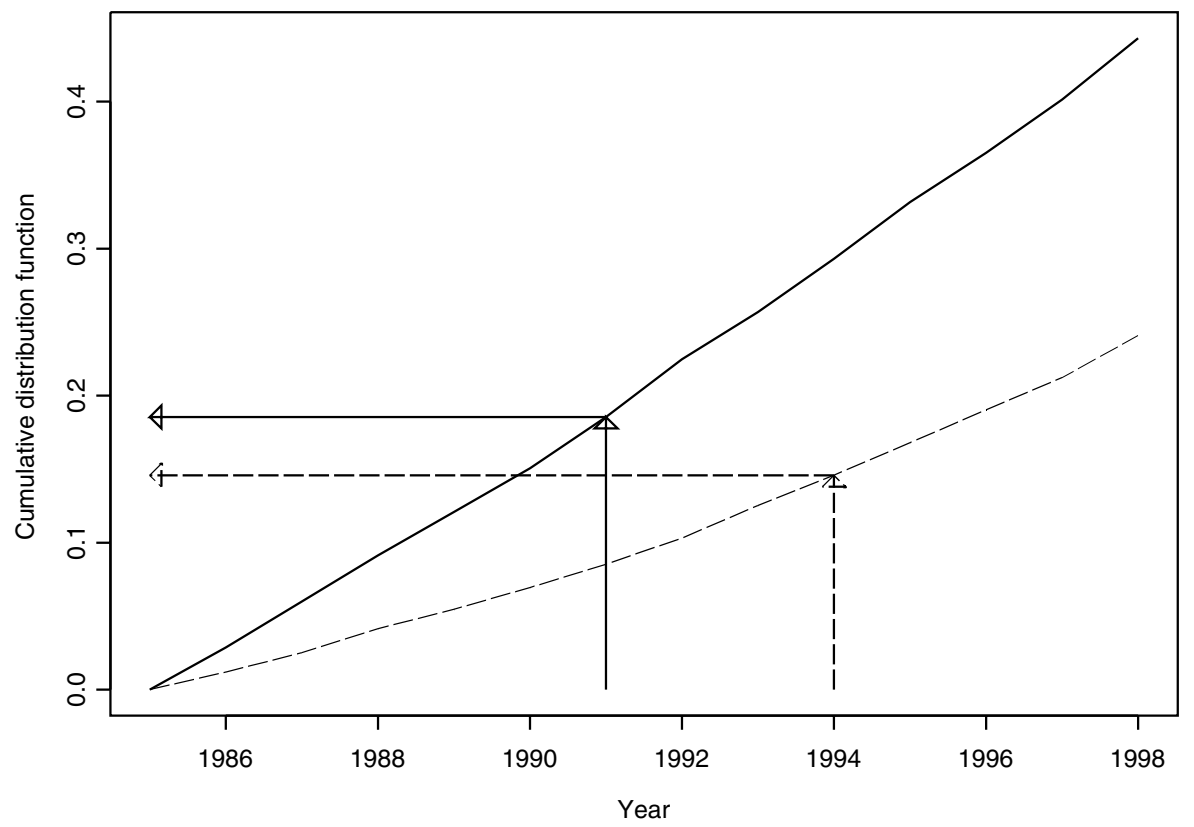

Fig. 2. Cumulative distribution functions for the residual lifetimes of men ( - ) and women $(---)$ aged 63 years in 1986: $\longrightarrow,--\longrightarrow$, computation of the outcome measure

rates apply. These expected probabilities are then compared with the observed survival. A statement that relative survival of a group of patients after 1 year of follow up was $r(1)=0.8$ then means that the surviving proportion of patients was 0.8 of the proportion that we would have expected on the basis of population tables. Such information is very useful, in particular in studies that aim to estimate cause-specific mortality but do not have good information on the cause of death. However, these methods focus on group experience and say nothing about individual values. For example, they do not answer the very natural question 'How long, relative to the general population, has a certain person lived?'. Or 'What proportion of patients lived longer than their expected medians (or quartiles, deciles, ...)?'.

Let us take another look at Fig. 2, the CDFs for patients aged 63 years in 1986. Assume that we were observing a man who subsequently died in 1991 at age 68 years and a woman who died in 1994 at age 71 years. Although the woman lived 3 years longer, relative to the background population her residual lifetime was surprisingly shorter than that of the man, as her value on the CDF achieved at age 71 years is lower than the value that was achieved by the man on his $\mathrm{CDF}$.

This leads to a fundamental observation: what is comparable is the value achieved on the expected residual CDF. Denote the background population residual lifetime CDF for a given age, sex and cohort year by $F_{A S Y}$. Then the quantity that we are interested in is the transformation $y=F_{A S Y}(t)$ which converts the actual survival time $t$ to the associated value $y$ on the CDF. It then makes sense to take as an outcome measure the random variable $Y=F_{A S Y}(T)$, which measures how long, after the event, and relative to the respective population, a person has lived. If $T$ is censored, so is $Y$. For any given $t$, age, sex and cohort year, the respective $y$ is calculated from population tables. Of course, as population tables are given in yearly intervals, a simple interpolation is necessary. 


\section{Properties of the outcome measure}

Two properties of the outcome measure are immediate.

(a) Given equal values of age, sex and cohort year, $Y$ increases monotonically with $T$ and ordering is preserved.

(b) When subjects with different age, sex and cohort years but the same survival time $T$ are compared, the transformed value is larger for the person who has been exposed to and survived the greater cumulative population hazard $\Lambda(T)$ (has done better against his or her odds). This is automatic from the definition $S(t)=\exp \{-\Lambda(t)\}$ so the ranking of two values $\Lambda_{A S Y_{1}}(t)$ and $\Lambda_{A S Y_{2}}(t)$ is the same as the ranking of $y_{1}=1-S_{A S Y_{1}}(t)$ and $y_{2}=1-S_{A S Y_{2}}(t)$, which represent the values of our measure.

Although these requirements are necessary if the outcome measure is to make sense, we can deduce other properties, that are not immediately obvious, which strengthen our belief in the usefulness of the approach. First note that, although $Y$ is a probability and conditioned to lie in $(0,1)$, standard survival methods can still be used in summarizing the observed, possibly censored, values.

(c) From the probability integral transform, $Y$ is uniformly distributed if background rates apply and $Y=F_{A S Y}(T)$ is the true CDF. This means that the expected (population) survival function of $Y$ is simply $S_{Y}(y)=1-y$. If the group under study does worse than the population, then the estimated survival function for $Y$ will go below this straight line; if better, above. Fig. 3 gives the Kaplan-Meier curve for our AMI example, using all patients combined. The vertical and horizontal lines illustrate the type of information that can be read from such a plot. Starting at $Y=0.5$, we see that an estimated proportion of $34 \%$

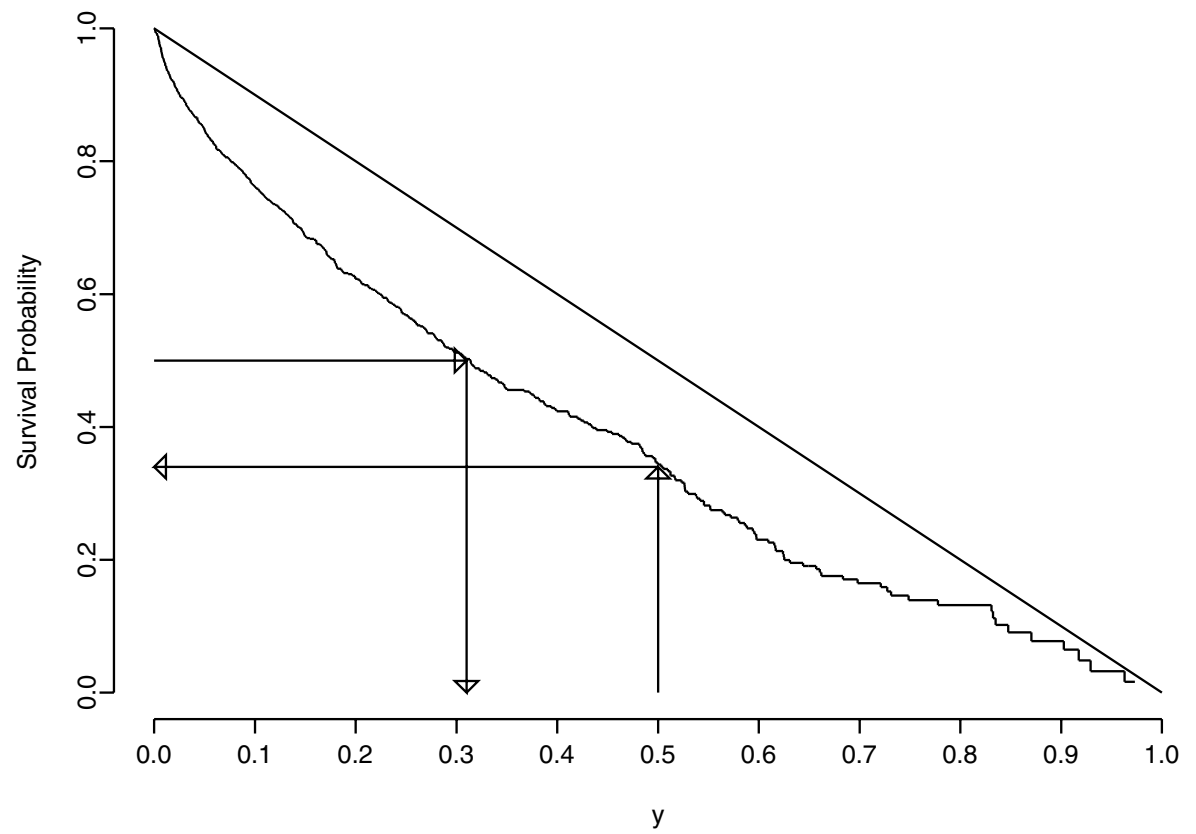

Fig. 3. Kaplan-Meier survival estimate for all patients with $Y$ as outcome: $\backslash$, expected survival function under the null hypothesis that background rates adjusted for age, sex and cohort year apply; $\longrightarrow$, illustration of how additional information can be obtained (see the text for details) 


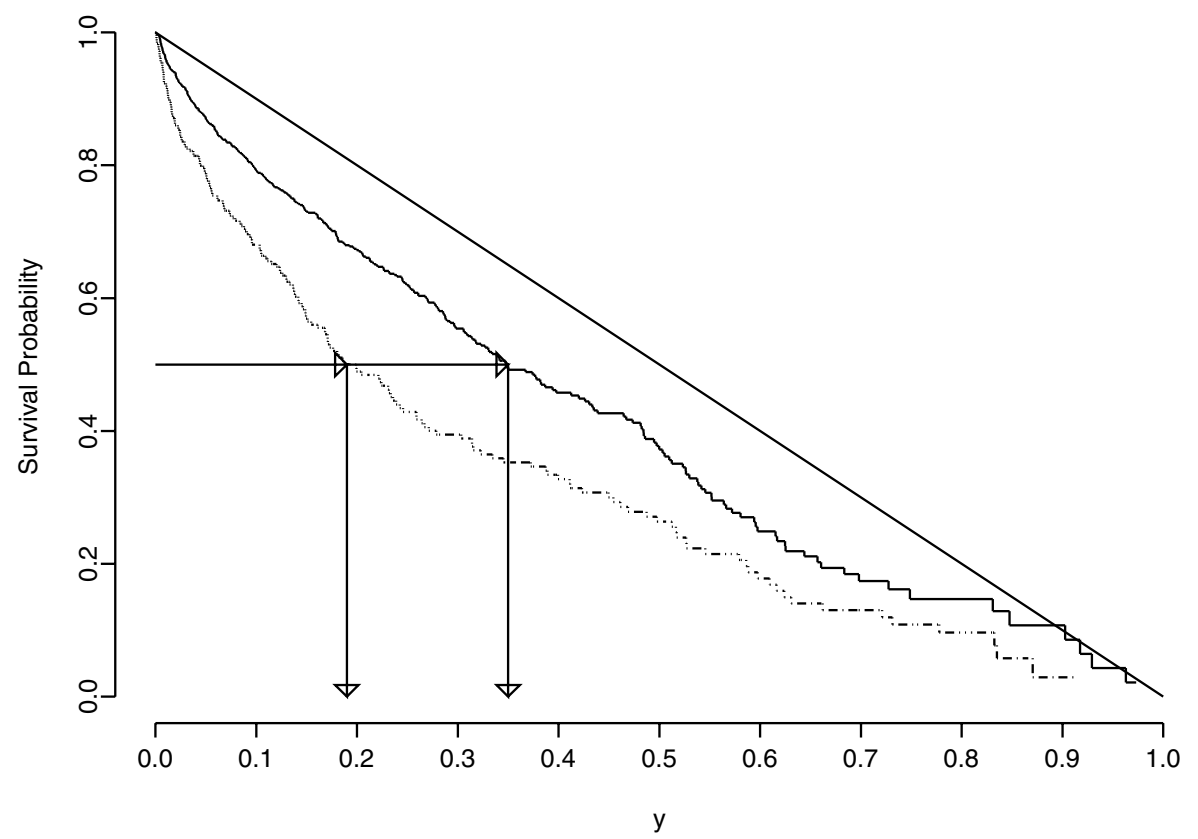

Fig. 4. Kaplan-Meier survival estimates for men $(\neg)$ and women ( $\left.{ }^{\cdots}{ }^{\circ} \mid . ..\right)$ with $Y$ as the outcome

of patients lived longer than the medians in their respective populations. In the other direction, starting at survival 0.5 , we conclude that the observed median corresponds to the 31 st percentile in the background populations. We could of course do this for any quantile. Such information is not obtainable from standard relative survival methods.

(d) The hazard of the general population can be (and often is) thought of as the base-line hazard. From point (c) above it immediately follows that in terms of $Y$ the base-line hazard has the form $1 /(1-y)$ under the null hypothesis of no difference from background. We can test this by using standard techniques. For our example in Fig. 3, a Kolmogorov test for censored data (Guilbaud, 1988) shows highly significant $(p<0.0001)$ differences between the observed and background survival functions.

(e) Similarly we can use standard survival techniques for group comparisons after transformation, without the need for specialist software. Fig. 4 shows Kaplan-Meier estimates for men and women by using $Y$ as the outcome. The graph tells a similar story to that of Fig. 1 but we can now test for a differential sex effect by using a simple log-rank test, giving $p<0.0001$ and confirming that the relative survival of women after AMI is worse than that of men. Additionally, the arrows show that half of the women died before their expected 19th percentile, whereas half of the men died before their 35th percentile. Turning to the age effect, Fig. 5 shows Kaplan-Meier curves for two age groups (below and above the median) for both the original time variable $T$ and the transformed variable $Y$. We see that, at least looking at it univariately, no excess risk is associated with age.

The numbers in Table 3 represent $y$ for various combinations of age, sex and survival time, calculated under the assumption that all observations started in 1983 . We call the reader's attention to the fact that a woman who is 70 years old and who survived 5 years lived relatively longer than a woman who is 50 years old and who survived 15 years, but the reverse is true for men. 


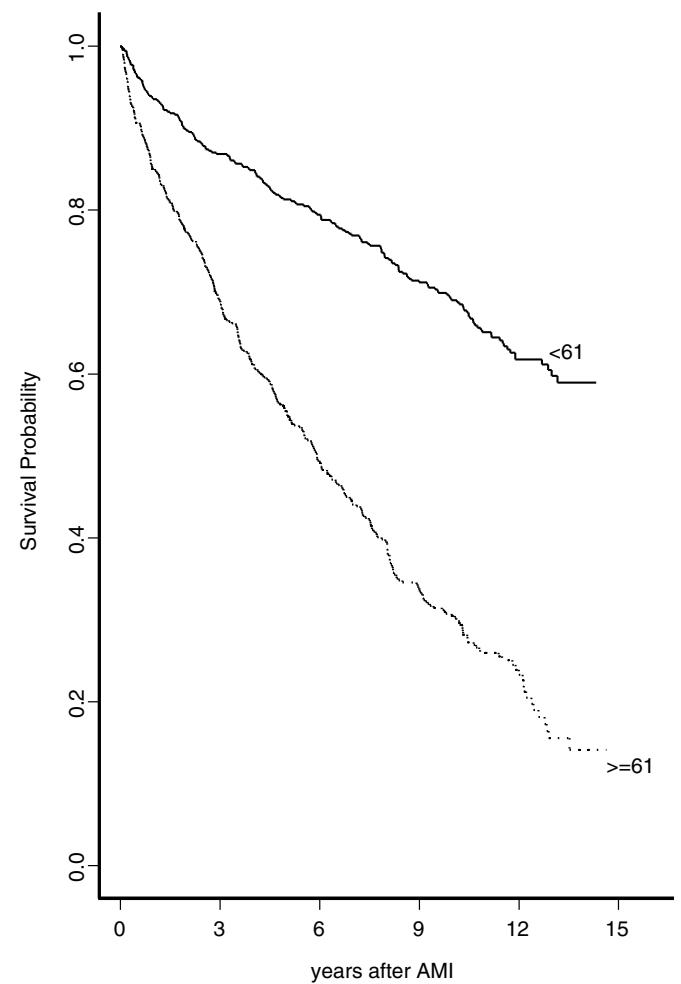

(a)

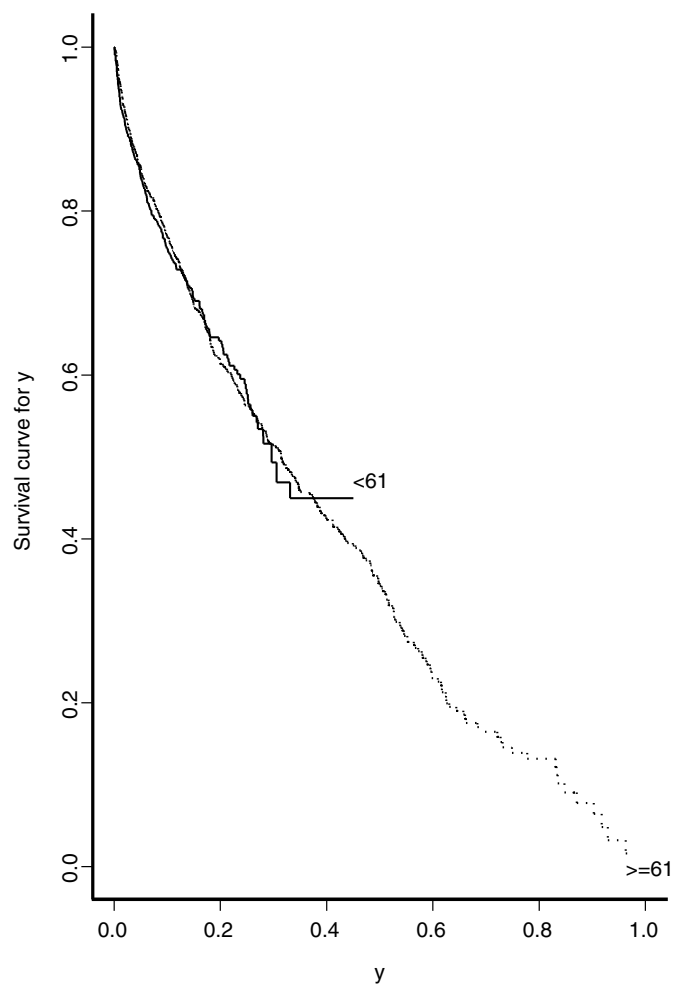

(b)

Fig. 5. Kaplan-Meier survival estimates for (a) the original time and (b) the transformed variable, stratified by age group $(-,<61$ years; $\cdots \cdots, \geqslant 61$ years): the effect of age disappears after transformation

Table 3. Four examples of calculating $y$ for different sex, age and durations of survival

\begin{tabular}{|lll|}
\hline \multirow{2}{*}{ Lived (years) } & \multicolumn{2}{c|}{$y$} \\
& Men & Women \\
\cline { 2 - 3 } & & \\
& & \\
$50-65$ & 0.25 & 0.10 \\
$60-75$ & 0.45 & 0.23 \\
$70-75$ & 0.24 & 0.15 \\
$75-90$ & 0.91 & 0.82 \\
& & \\
\hline
\end{tabular}

The final point in this section is that transforming to $Y$ removes expected survival differences due to age, sex and cohort year. Any differences that remain must of course be attributed to differential risk associated with the event of interest. If there is no differential risk, we shall see no differences in the new outcome. Often there will be increased risk (see Section 5), often named the excess risk, compared with the background, but our method is also appropriate if risk is reduced, e.g. if the study is into the effects of health education. 


\section{Regression}

$Y$ is a non-negative, ratio level, numerical variable, censored as is the original time, and can be modelled as such by using the usual survival analysis machinery and standard survival software. Any method can be used, although the choice might often fall on the Cox model. Covariates other than age, sex and cohort year can of course be included in such models.

Usually, population tables will not be broken down by other variables (race is a possible exception), meaning that coefficients for such variables cannot be interpreted in terms of excess risk. In fact, in the Cox model, we should not expect them to change at all. To show this we first express the survival function for $Y$ in terms of a survival function for $T$, using obvious notation

$$
S_{Y}(y)=P(Y>y)=P\left\{T>F_{A S Y}^{-1}(y)\right\}=S_{T}\left\{F_{A S Y}^{-1}(y)\right\} .
$$

Now introduce covariates and write $c$ for those for which separate population data are available (e.g. age, sex and cohort year), $x$ for the remainder of interest and $z$ for the combined set $(c, x)$. Assume that the hazard for $T$ follows a Cox proportional hazards model

$$
S_{T}(t)=\exp \left\{-\Lambda_{0 T}(t) \exp (\gamma c+\beta x)\right\} .
$$

Then since

$$
S_{Y}(y)=\exp \left\{-\Lambda_{Y}(y)\right\}
$$

we must have

$$
\Lambda_{Y}(y)=\Lambda_{0 T}\left\{F_{A S Y}^{-1}(y)\right\} \exp (\gamma c+\beta x) .
$$

Incorporating the regression effects of $c$ into the base-line hazard we can write

$$
\Lambda_{Y}(y)=\Lambda_{0 c}(y) \exp (\beta x)
$$

for suitable base-line cumulative hazards $\Lambda_{0 c}$. This is a stratified Cox model for $Y$, with a different base-line for each age-sex-year cohort $c$, but with the same regression effect for other covariates $x$. Hence we expect estimates of $\beta$ for proportional hazards analyses of either $Y$ or $T$ to be close.

Returning to our example on AMI, Tables 4 and 5 give the results of a Cox regression for the untransformed and transformed time. Besides age, sex and cohort year, we have eight additional dichotomous variables in the model, coded 1 to denote the presence of the factor and 0 to denote the absence of the factor.

As expected, there is no real change in the estimated coefficients for the variables that do not enter the population table, nor for cohort year, since the population tables do not change much in 5 years. There is quite a change for the sex and age coefficients, however. The effect of sex is the same as in the univariate analysis that was shown earlier: sex does not significantly influence actual survival after AMI, but the transformed model shows a greater relative loss for women. For age, from the first model we conclude that higher age means higher risk, which is of course to be expected when the event is death from any cause. However, we read from the relative survival model results in Table 4 that older age means losing relatively less. Recall that there was no marginal effect of age on relative survival (see Fig. 5) and the result in Table 4 shows the importance of taking all variables into account. Older subjects have more risk factors present (a higher percentage of reinfarctions, diabetes, hypertension etc.), which mask the age effect in the marginal analysis. Once we have controlled for them, we see that the relative residual lifetime for older subjects is higher than for younger subjects. This interesting result could be because younger people who suffer early AMI are in some way more frail relative to the background 
Table 4. Cox model fit to untransformed AMI data

\begin{tabular}{|lrccrc|}
\hline Variable & coef & $\exp ($ coef $)$ & se(coef) & $z$ & $p$ \\
\hline age & 0.049 & 1.05 & 0.005 & 10.75 & 0.000 \\
sex & -0.078 & 0.93 & 0.104 & -0.76 & 0.450 \\
cohort year & -0.112 & 0.89 & 0.036 & -3.08 & 0.002 \\
arrhythmia & 0.317 & 1.37 & 0.097 & 3.26 & 0.001 \\
insufficiency & 0.498 & 1.65 & 0.102 & 4.90 & 0.000 \\
hypertension & 0.311 & 1.37 & 0.092 & 3.39 & 0.001 \\
diabetes & 0.472 & 1.60 & 0.105 & 4.50 & 0.000 \\
aspirin & -0.254 & 0.78 & 0.097 & -2.62 & 0.009 \\
beta blocker & -0.323 & 0.72 & 0.115 & -2.82 & 0.005 \\
other medicine & 0.241 & 1.27 & 0.094 & 2.55 & 0.011 \\
reinfarction & 0.372 & 1.45 & 0.127 & 2.93 & 0.003 \\
\hline
\end{tabular}

Table 5. Cox model fit to transformed AMI data

\begin{tabular}{|lrccrc|}
\hline Variable & coef & exp (coef) & se(coef) & $z$ & $p$ \\
\hline age & -0.035 & 0.97 & 0.005 & -6.38 & 0.000 \\
sex & 0.440 & 1.55 & 0.109 & 4.05 & 0.000 \\
cohort year & -0.096 & 0.91 & 0.036 & -2.68 & 0.007 \\
arrhythmia & 0.323 & 1.38 & 0.097 & 3.31 & 0.001 \\
insufficiency & 0.517 & 1.68 & 0.102 & 5.08 & 0.000 \\
hypertension & 0.337 & 1.40 & 0.092 & 3.65 & 0.000 \\
diabetes & 0.479 & 1.62 & 0.105 & 4.57 & 0.000 \\
aspirin & -0.250 & 0.78 & 0.097 & -2.57 & 0.010 \\
beta blocker & -0.332 & 0.72 & 0.115 & -2.89 & 0.004 \\
other medicine & 0.250 & 1.29 & 0.094 & 2.65 & 0.008 \\
reinfarction & 0.373 & 1.45 & 0.127 & 2.93 & 0.003 \\
\hline
\end{tabular}

population, whereas elderly patients with or without AMI are more comparable. Dickman et al. (2004) found a similar result in a survival study of patients with skin melanoma. After a high risk period following diagnosis, the most elderly patients had the best subsequent relative survival, a possible explanation being that elderly patients who survive the high risk period are naturally more robust. The AMI data include only patients who were discharged from hospital and therefore also survived a high risk period after infarction, so a similar point could apply. Aalen (1994) discussed the effect of selection effects in survival studies due to unmeasured frailty.

\subsection{Extensions}

Given that in our approach we have a direct individual level measure of relative survival before we attempt modelling, further standard survival techniques are immediately available. For instance, if we assume a Cox proportional hazards model, the armoury of diagnostic techniques can be applied as usual. Also, standard extensions of the basic Cox model (Therneau and Grambsch, 2000) can be considered. For instance, time-dependent covariates can easily be incorporated, after the actual times have been transformed into the scale of $Y$.

To repeat, we have discussed a Cox proportional hazards model for the transformed survival time but other options are available, provided that the restriction of $Y$ to $(0,1)$ is retained. For 
example we might consider relating the observed survival function to the population base-line through a power law model

$$
S_{Y}(y)=(1-y)^{\alpha \exp (\beta z)} \quad 0 \leqslant y \leqslant 1
$$

which is a special case of proportional hazards with base-line known up to the parameter $\alpha$, and which can be fitted by maximum likelihood. Alternatively, standard models can be specified for the cumulative population hazard transformation $\Lambda=-\log (1-Y)$, which takes values on the positive half-line.

One purpose of modelling, whichever model is selected, is for prediction. Again this causes no difficulty, remembering that predicting values of $Y$ means predicting quantiles of the conditional distribution in the population subgroup. If we want to transform these into actual time, we simply reverse the transformation procedure that led to values of $Y$.

\section{Relationship to other regression approaches}

Several approaches to regression for relative survival have been proposed in the literature (see for example Andersen et al. (1985), Hakulinen and Tenkanen (1987), Andersen and Væth (1989) or Estève et al. (1990)). Basically we can distinguish two types of model: additive and multiplicative for hazards. In this section we discuss the two types of model and give a comparison between the Cox model in $Y$ and the multiplicative model of Andersen et al. (1985).

\subsection{Additive hazard models}

Under the additive model the observed hazard is a sum of the population hazard and a nonnegative excess term

$$
\lambda_{\mathrm{O}}(t, z)=\lambda_{\mathrm{P}}(t, c)+\lambda_{\mathrm{E}}(t, z)
$$

where $z=(c, x)$ and $c$ denotes the variables by which population values are stratified. This gives a multiplicative relationship for the survival functions

$$
S_{\mathrm{O}}(t, z)=S_{\mathrm{P}}(t, c) r(t, z)
$$

where $r(t, z)=\exp \left\{-\int_{0}^{t} \lambda_{\mathrm{E}}(u) \mathrm{d} u\right\}$. Hence the phrase 'relative survival model' is sometimes used specifically for the additive hazard class, whereas in our sense the term means any model which relates observed survival to the background. By assumption $\lambda_{\mathrm{O}}(t, z) \geqslant \lambda_{\mathrm{P}}(t, z)$ at all times and for any values of covariates, and $r(t, z)$ is a proper survival function. This will often be true, especially in cancer research. However, there can be subgroups of subjects who do better than the population, in which case the model would not be a good choice.

A variety of methods have been proposed for fitting the additive hazard regression model. Dickman et al. (2004) described and compared four, assuming constant excess hazard in prespecified time bands. Their finding is that the methods of Estève et al. (1990) and of Hakulinen and Tenkanen (1987), although conceptually different (individual versus grouped data), are essentially equivalent, and that for practical purposes maximum likelihood estimation under a generalized linear model with Poisson error structure and a user-defined link function works well and moreover can be implemented in standard software.

A similar additive structure might be taken for our outcome measure $Y$,

$$
\lambda_{\mathrm{O}}(y, z)=\frac{1}{1-y}+\lambda_{\mathrm{E}}(y, z),
$$


with $\lambda_{\mathrm{E}}(y, z)$ perhaps assumed to be constant in $y$ over specified bands, deciles for instance. There is no simple relationship between this model and the proportional hazards model for $Y$ that was discussed in the previous section.

\subsection{Multiplicative models}

A general multiplicative hazard model is

$$
\lambda_{\mathrm{O}}(t, z)=\lambda_{\mathrm{P}}(t, c) \nu(t, z) .
$$

The unit-free factor $\nu(t, z)$ can be seen as relative mortality and for this reason models of this type are sometimes called relative mortality models. The model has fewer mathematical restrictions than the additive model (1) but there have been arguments that additive models can be more realistic in practice, for cancer studies at least (Dickman et al., 2004; Buckley, 1984). How well model (2) will fit depends of course on what we assume for $\nu(t, z)$ and here we shall concentrate on the Andersen et al. (1985) form, which can be written as

$$
\lambda_{T}(t, z)=\nu_{0}(t) \lambda_{A S Y}(t) \exp \left(\gamma_{T} c+\beta_{T} x\right),
$$

where we use the subscript $T$ to be clear which outcome we have in mind, $\nu_{0}(t)$ is an unspecified base-line relative mortality and $\lambda_{A S Y}(t)$ is the appropriate population mortality. Fitting is straightforward by including population mortality rates as a time-dependent covariate in the Cox model.

As shown above, assuming that we have proportional hazards in $Y$, it follows that we need not have proportional hazards in $T$ and hence the approach of Andersen et al. (1985) will in general not give identical results to those of an analysis of $Y$. It may be instructive therefore to see where the differences can come from. One can show that, if equation (3) is true, the hazard function for the transformed variable $Y$ can be expressed as

$$
\lambda_{Y}(y, z)=\nu_{0}\left\{F_{A S Y}^{-1}(y)\right\} \frac{1}{1-y} \exp \left(\gamma_{T} c+\beta_{T} x\right) .
$$

Whereas covariates $x$ retain the proportional structure, as shown in Section 4, the inclusion of the term $\nu_{0}\left\{F_{A S Y}^{-1}(y)\right\}$ will affect the estimation of the $\gamma$-coefficients, though not in any simple way. Of course, in the special case $\nu_{0}(t)=1$, when model (3) becomes the Breslow model (Breslow et al., 1983), the proportionality is preserved. As noted in Section 3, the base-line hazard in this case becomes $1 /(1-y)$.

Interestingly, Cox model fits to the AMI data gave very similar results, suggesting that in this case a transformation via expected CDFs preserves proportionality. Simulation studies, which are not reported, show that results under the two approaches can sometimes be markedly different.

\section{Discussion}

For regression problems, the standard additive and multiplicative approaches have one fundamental similarity: they both model $\lambda_{T}(t, z)$ while controlling for population differences in mortality. In contrast, our proposal is to model $\lambda_{Y}(y, z)$ after adjusting for expected survival and hence we explicitly separate the two issues. A possible disadvantage is that the interpretation of results after a change to a new scale may be seen as less straightforward than for direct hazard models. The real question when selecting a model of course is whether it is appropriate for the data, and here further work on model selection techniques for relative survival would be useful. 
We described an efficient, yet technically simple, way to analyse relative survival data. The method is intuitively appealing, and its implementation requires no special software. Apart from a simple calculation of expected CDFs, requiring multiplication of relevant entries in population mortality tables, the standard apparatus of survival programs in most packages will suffice. But, more importantly, our method sheds a new light on relative survival. First, by calculating the proportion of patients who survived longer than a given quantile of the respective general population, we obtain some new information about the relative survival. Second, the comparison between the sample and population is different from those of the standard methods, and testing for group differences is easy. Third, the usage of regression methodology, in particular the Cox model, is straightforward but not equivalent to other known methods. In short, our methods complement the standard methods and add to the richness of the relative survival methodology.

\section{Acknowledgements}

We are grateful for the helpful comments of the Joint Editor, Associate Editor and two referees.

\section{References}

Aalen, O. O. (1994) Effects of frailty in survival analysis. Statist. Meth. Med. Res., 3, 227-243.

Andersen, P. K., Borch-Johnsen, K., Deckert, T., Green, A., Hougaard, P., Keiding, N. and Kreiner, S. (1985) A Cox regression model for relative mortality and its application to diabetes mellitus survival data. Biometrics, 41, 921-932.

Andersen, P. K. and Væth, M. (1989) Simple parametric and nonparametric models for excess and relative mortality. Biometrics, 45, 523-535.

Breslow, N. E., Lubin, J. H., Marek, P. and Langholz, B. (1983) Multiplicative models and cohort analysis. J. Am. Statist. Ass., 78, 1-12.

Buckley, J. D. (1984) Additive and multiplicative models for relative survival rates. Biometrics, 40, 51-62.

Dickman, P. W., Sloggett, A., Hills, M. and Hakulinen, T. (2004) Regression models for relative survival. Statist. Med., 23, 51-64.

Ederer, F., Axtell, L. M. and Cutler, S. J. (1961) The relative survival rate: a statistical methodology. Natn. Cancer Inst. Monogr., 6, 101-121.

Ederer, F. and Heise, H. (1959) Instructions to IBM 650 programmers in processing survival computations. Methodological Note 10, end results evaluation section. National Cancer Institute, Bethesda.

Estève, J., Benhamou, E., Croasdale, M. and Raymond, M. (1990) Relative survival and the estimation of net survival: elements for further discussion. Statist. Med., 9, 529-538.

Guilbaud, O. (1988) Exact Kolmogorov-Smirnov tests for left-truncated and/or right censored data. J. Am. Statist. Ass., 83, 213-221.

Hakulinen, T. (1982) Cancer survival corrected for heterogeneity in patient withdrawal. Biometrics, 38, 933-942.

Hakulinen, T. and Tenkanen, L. (1987) Regression analysis of relative survival rates. Appl. Statist., 36, $309-317$.

Therneau, T. M. and Grambsch, P. M. (2000) Modeling Survival Data: Extending the Cox Model. New York: Springer. 